\title{
DETERMINANTES DO BAIXO PESO AO NASCER A PARTIR DAS DECLARAÇÕES DE NASCIDOS VIVOS
}

\author{
LOW BIRTH WEIGHT DETERMINANTS PRESENT IN THE \\ STATEMENT OF LIVE BIRTH
}

\section{DETERMINANTES DEL BAJO PESO AL NACER PRESENTES EN LA DECLARACIÓN DE NACIDO VIVO}

\author{
Giselle Mesquita Costa* \\ Jacqueline Domingues Tibúrcio ** \\ Valéria Conceição de Oliveira ${ }^{* * *}$ \\ TARCísio LaERTE GonTijo ${ }^{* * * *}$ \\ Eliete Albano de Azevedo Guimarães ${ }^{* * * *}$
}

\begin{abstract}
RESUMO
O objetivo desse estudo foi identificar a prevalência e os fatores associados ao baixo peso ao nascer (PBN) em Divinópolis, Brasil. Método: Trata-se de estudo transversal que analisou 9.987 registros de nascidos vivos hospitalares e únicos ocorridos entre 2008 e 2011. Na análise univariada utilizou-se o teste qui-quadrado e a razão de chances fixando um nível de significância de 5\%. Para o controle de possíveis fatores de confusão nas associações obtidas, usou-se o modelo de regressão logística binária. Resultados: A tendência do BPN no período estudado mostrou-se estável, variando entre 8,9 e 9,2\%. Mais da metade das crianças nasceu prematuro (62,0\%) e de parto cirúrgico $(63,9 \%)$. Os fatores independentes foram: prematuridade, sexo feminino, realização de seis ou menos consultas de pré-natal, mães solteiras. Conclusão: O conhecimento dos determinantes do BPN permite aos profissionais de saúde organizar a rede assistencial na proporção da necessidade e para aqueles em situação de maior vulnerabilidade e risco de agravos à saúde.
\end{abstract}

Palavras chave: Recém-nascido de baixo peso, enfermagem materno-infantil, sistemas de informação em saúde, fatores epidemiológicos.

\begin{abstract}
The aim of this study was to identify the prevalence and the low birth weight (LBW) associated factors in Divinópolis, Brazil. Method: It is a cross-sectional study. 9,987 hospitalar birth records occurred between 2008 and 2011, were analysed. In univariate analysis, chi-square test and the odds ratio was used setting a significance

\footnotetext{
* Acadêmica de Enfermagem da Universidade Federal de São João Del Rei. Divinópolis, Brasil. E-mail: gisa_mesquita@ hotmail.com

${ }^{* *}$ Estatística. Professora da Universidade Federal de São João Del Rei. Divinópolis, Brasil. E-mail: jacqueline_tiburcio@ufsj. edu.br

${ }^{* * *}$ Enfermeira. Professora da Universidade Federal de São João Del Rei. Divinópolis, Brasil. E-mail: valeria.oli.enf@gmail.com

${ }^{* * * *}$ Enfermeiro. Professor da Universidade Federal de São João Del Rei. Divinópolis, Brasil. E-mail: enftarcisio@yahoo.com.br

${ }^{* * * * *}$ Enfermeira. Professora da Universidade Federal de São João Del Rei. Divinópolis, Brasil. E-mail: elietealbano@ufsj.edu.br
} 
level of 5\%. To control for possible confounding factors in obtained associations, binary logistic regression model was used. Results: The LBW trend during studied period was stable, ranging between 8.9 and 9.2\%. More than half of children were born preterm (62.0\%) and through operative delivery (63.9\%). Independent predictors were prematurity, female, performing six or fewer prenatal visits, single mothers. Conclusion: The knowledge of the determinants of LBW allows health professionals to organize health care network in proportion to the need for those most vulnerable and in risk of health problems.

Key words: Infant, low birth weight, maternal child nursing, health information systems, epidemiologic factors.

\section{RESUMEN}

El objetivo de este estudio fue identificar la prevalencia y los factores asociados con el bajo peso al nacer (BPN) en Divinópolis, Brasil. Material y Método: Se trata de estudio transversal que analizó 9.987 registros de nacimientos individuales vivos que ocurrieron en hospitales entre 2008 y 2011. En el análisis univariado se utilizó la prueba de chi-cuadrado y odds ratio estableciendo un nivel de significación del 5\%. Para controlar los posibles factores de confusión en las asociaciones obtenidas, se utilizó el modelo de regresión logística binaria. Resultados: La tendencia de BPN durante el período de estudio se mantuvo estable, oscilando entre el 8,9 y el 9,2\%. Más de la mitad de los niños con bajo peso nació prematuro $(62,0 \%)$ y a través de parto quirúrgico $(63,9 \%)$. Los predictores independientes fueron la prematuridad, sexo femenino, realizar seis o menos consultas prenatales, madres solteras. Conclusión: El conocimiento de los factores determinantes permite a los profesionales de salud organizar una red de atención de salud proporcional a las necesidades de aquéllos en situación de mayor vulnerabilidad y riesgo de problemas de salud.

Palabras clave: Recién nacido de bajo peso, enfermería materno-infantil, sistemas de información en salud, factores epidemiológicos.

Fecha recepción: 08/11/13. Fecha aceptación: 11/10/14.

\section{INTRODUÇÃO}

$\mathrm{O}$ enfrentamento da mortalidade infantil coloca-se atualmente como uma das prioridades da política pública de saúde em todas as instâncias de gestão e assistência. No campo da saúde, o Brasil já cumpriu um dos objetivos da primeira Meta de Desenvolvimento para o Milênio (redução no número de crianças subnutridas), estabelecida pela Organização das Nações Unidas e está caminhando para o cumprimento da quarta meta que é a redução de dois terços na taxa de mortalidade de crianças menores de 5 anos (1). Nas últimas três décadas, a mortalidade infantil no Brasil vem diminuindo, cerca de $6,3 \%$ ao ano (2).
Dentre as principais causas de mortalidade infantil, destaca-se o baixo peso ao nascer (BPN), definido como o nascimento de uma criança com peso inferior a 2.500 gramas. $\mathrm{O}$ encurtamento da gestação (prematuridade) e a restrição do crescimento fetal (retardo de crescimento intra uterino - CIUR) são os fatores, que isolados ou associados, resultam em nascimentos com baixo peso (3).

Anualmente nascem cerca de 20 milhões de crianças com BPN em todo mundo, representando cerca de $15,5 \%$ de todos nascimentos (4). As prevalências do BPN variam em cada país atingindo valores que vão de e $7,0 \%$ nos países desenvolvidos a $16,5 \%$ nos países em desenvolvimento. A Ásia e África juntos são responsáveis por cerca de $87 \%$ dos nascimentos de baixo peso no mundo, sen- 
do somente a Índia, responsável por 39\%. Na América do Sul a média de nascimentos com baixo peso ao nascer é de 9,6\%, variando desde $5 \%$ no Chile a 16\% no Equador. Essa média é considerada alta quando comparada aos países da Europa e América do Norte que apresentam média de $6,4 \%$ e $7,7 \%$, respectivamente $(5,6)$.

No Brasil, em 2010 a prevalência foi de $8,4 \%$, variando de acordo com as regiões geográficas entre 7,3 e 9,2\%, respectivamente nas regiões Norte e Sudeste. Em Minas Gerais, no mesmo ano, o BPN foi 9,5\% do total de nascimentos, e em suas macrorregiões de saúde foram observadas variações de $8,4 \%$ (Jequitinhonha) a 10,5\% (Centro Sul) (7).

Destacam-se como fatores desencadeantes do BPN, entre outros, as condições socioeconômicas, o peso da mãe antes e durante a gestação, a idade e escolaridade materna, a duração da gestação, o tipo de parto, a paridade, a história obstétrica anterior, os cuidados pré-natais, a morbidade materna durante a gravidez e o tabagismo (3, 8-11).

Assim, avaliar a magnitude desse evento e de seus determinantes é essencial para a instituição de políticas públicas de saúde e nutrição, e para o planejamento do cuidado materno-infantil (10).

$\mathrm{O}$ acesso a essas e outras informações sobre as condições de nascimento permite integrar os profissionais, dentre eles os enfermeiros, instrumentalizando-os para a programação de ações e práticas em saúde, sobretudo na atenção primária, pela proximidade com o cotidiano das famílias e suas necessidades (12).

Muitas das informações produzidas para monitorar e avaliar a prevalência do BPN e de outros indicadores relativos ao recémnascido, à gestação, ao parto e às características da mãe encontram-se na Declaração de Nascido Vivo (DNV), documento oficial, individualizado, utilizado para a coleta dos dados sobre os nascimentos e para a lavratura da Certidão de Nascimento (13). Essas informações alimentam a base de dados do Siste- ma de Informação sobre Nascidos Vivos (SINASC) que quando utilizadas pelos gestores, profissionais de saúde e usuários permitem a realização de atividades gerenciais efetivas na implantação de ações e serviços que resultem em condições ideais de nascimento $(12,14)$.

A apropriação do conhecimento sobre a distribuição do BPN e seus determinantes influencia o poder decisório segundo a necessidade e a situação de maior vulnerabilidade e risco de agravos à saúde materno-infantil, garantindo assim, o princípio da equidade estabelecido no Sistema Único de Saúde (SUS). Sabendo-se que os determinantes do BPN possuem uma distribuição diferenciada e específica para populações distintas, em função principalmente das condições de vida, este estudo, tem como objetivo identificar a prevalência e os fatores associados ao baixo peso ao nascer.

\section{MÉTODO}

Trata-se de um estudo transversal descritivo e analítico que consistiu na análise de todos os 10.266 nascimentos hospitalares, residentes no município de Divinópolis/MG, ocorridos entre 2008 e 2011. Destes, foram excluídos 279 nascimentos $(2,7 \%)$, por serem de partos gemelares. Foram analisados 9.987 registros de nascidos vivos hospitalares e únicos. O estudo transversal é um tipo de pesquisa em que a relação-exposição é avaliada, em uma determinada população, em um dado momento (15).

Os dados foram obtidos através da base de dados do SINASC, disponível na Secretaria Municipal de Saúde de Divinópolis. A fonte de dados do SINASC é a DNV, documento individualizado que deve ser preenchido para todos os nascimentos vivos ocorridos no país, hospitalares ou domiciliares e permite conhecer o número de nascimentos por município de ocorrência e residência e obter informações sobre as características da ges- 
tação e do parto, da mãe e do recém-nascido (13). Sobre a qualidade das informações do SINASC, estudos têm demonstrado maior cobertura do SINASC $(16,17)$, maior confiabilidade dos dados (18) e menor incompletude dos campos da DNV $(14,18,19)$, tornando-o uma fonte em potencial a ser utilizado no serviço e na academia.

Considerou-se como variável resposta o peso ao nascer, categorizada em baixo peso (recém-nascidos com peso inferior a 2.500 g), e peso adequado (maior ou igual a 2.500 g). As variáveis independentes (covariáveis) foram: sexo do recém-nascido (feminino e masculino); idade da mãe (menos de 15; 16 a 19; 20 a 34; mais de 35 anos); escolaridade da mãe (menos de 8 anos, 9 a 11, 12 anos e mais), duração da gestação categorizada em 36 ou menos (pré-termo) e 37 ou mais semanas (a termo); tipo de parto (vaginal e cesárea); número de consultas pré-natal (menos de 6 consultas e 7 e mais consultas), número de filhos tidos vivos em gestações anteriores e número de filhos nascidos mortos.

A análise da completude da DNV apontou para $100 \%$ de registro das variáveis, peso ao nascer, idade da mãe, tipo de parto, tipo de gravidez e sexo do recém-nascido. Nas demais variáveis estudadas, a incompletude variou de 0,2 a 1,8\%, classificação considerada excelente (menor de 5\%) (17). Em relação às demais variáveis que constam na DNV - estado civil da mãe, raça/cor da pele, ocupação materna e ramo de atividade, detecção de alguma má formação congênita e/ou anomalia cromossômica - não foram avaliadas devido a incompletudes maior de $20 \%$ na DNV, avaliadas como ruins (20).

Utilizou-se o software Statístical Package for the Social Sciences (SPSS ${ }^{\circledR}$ ) 17.0, para a tabulação e a análise dos dados. Para caracterização da população estudada foi realizada a distribuição de frequências e ou/medidas de tendência central e de dispersão das variáveis. O teste qui-quadrado foi realizado para avaliar possíveis associações entre as covariáveis e o BPN, fixando um nível de significância de
5\%. A força de associação foi estimada calculando-se a razão de chances (Odds Ratio) e seus intervalos de confiança de 95\% (IC95\%) para análise univariada. Para o controle de possíveis fatores de confusão nas associações obtidas, usou-se o modelo de regressão logística binária multivariada. O teste de Hosmer-Lemeshow foi empregado para verificar o ajuste do modelo. Foram introduzidas no modelo multivariado as variáveis que mostraram associação $(p$ valor $<0,20)$ com a variável dependente na análise univariada, permanecendo no modelo as variáveis significativas $(p$ valor $<0,05)$.

Este estudo obedeceu aos princípios éticos da Resolução 196/96 do Conselho Nacional de Saúde e foi aprovado pelo Comitê de Ética e Pesquisa da Fundação Educacional de Divinópolis/MG - FUNEDI, pelo Parecer No 036/2010.

\section{RESULTADOS}

Ao analisar a distribuição dos nascidos vivos segundo o peso ao nascer, identificouse que a curva tende à normalidade, com leve assimetria no sentido dos valores mais baixos (Gráfico 1). O peso médio entre os nascimentos analisados foi de $3.122 \mathrm{~g}$, desvio padrão $524 \mathrm{~g}$, e a prevalência do BPN no período analisado foi 9,0\% (897 neonatos). Destes, 56,1\% (502) foram pré-termo (menor de 37 semanas) e $393(43,9 \%)$ a termo (entre 37 e 42 semanas). Identificaram-se dois registros sem informação sobre a duração da gestação. A tendência do BPN entre 2008 e 2011 mostrou-se estável, variando de 8,9 a $9,2 \%$, e mantendo-se constante a partir do ano 2009 (Gráfico 2).

$\mathrm{Na}$ análise univariada identificou-se associação do BPN com a escolaridade materna, abalizando que quanto menor a instrução da mãe mais chances de ter nascimentos com baixo peso. Mães solteiras; mães com mais de 4 filhos nascidos vivos; mães com menos 
Gráfico 1. Distribuição do peso ao nascer de nascidos vivos hospitalares e únicos, Divinópolis, Minas Gerais, 2008 - 2011.

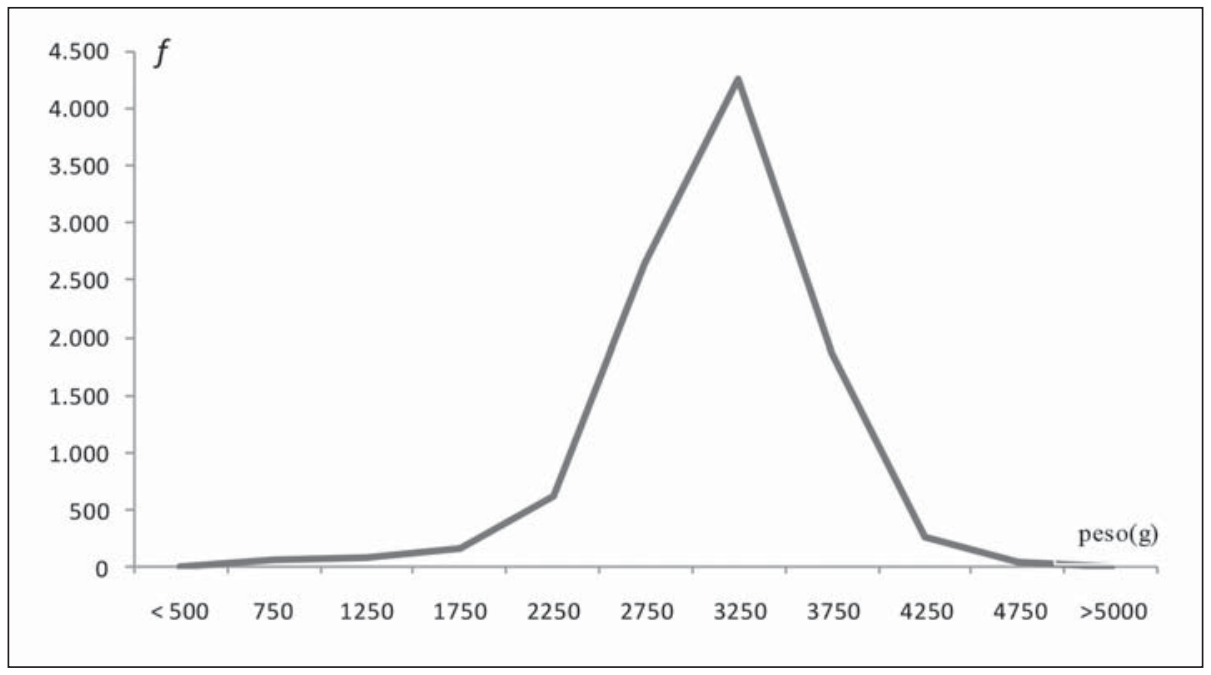

Gráfico 2. Prevalência de baixo peso ao nascer e intervalo de confiança de 95\%, Divinópolis, Minas Gerais, 2008 - 2011.

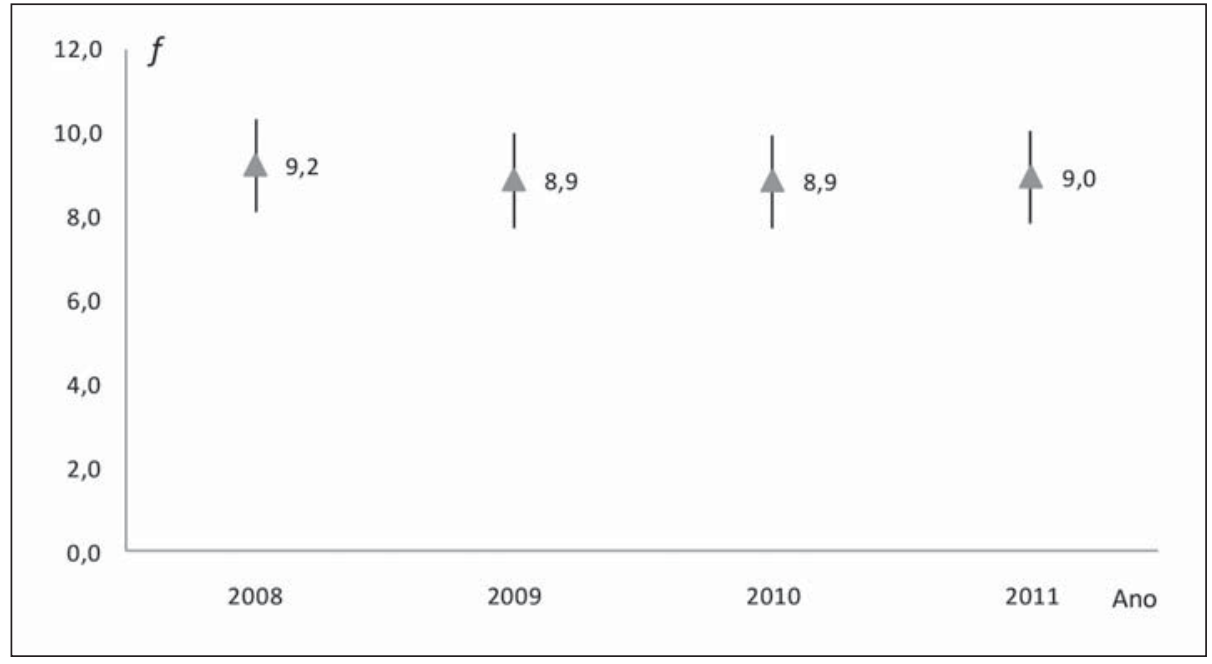

de 7 consultas de pré-natal durante a gestação; gestação com menos de 37 semanas; e recém-nascidos do sexo feminino também estiveram associados ao evento. As variáveis, tipo de parto, idade da mãe e número de filhos nascidos mortos não influenciaram o nascimento de recém-nascidos de baixo peso (Tabela 1).
A Tabela 2 apresenta os resultados do modelo final da análise multivariada para fatores independentes associados ao BPN. A ocorrência de BPN em Divinópolis/MG, no período analisado, esteve fortemente determinada pela prematuridade. Crianças prematuras nascidas com menos de 37 se- 
Tabela 1. Análise univariada para avaliar associação entre as covariáveis e o Baixo Peso ao Nascer, Divinópolis, Minas Gerais, entre 2008 e 2011.

\begin{tabular}{|c|c|c|c|c|}
\hline \multirow[t]{2}{*}{ Variáveis } & \multicolumn{2}{|c|}{ Baixo peso ao Nascer } & \multirow[t]{2}{*}{ OR $(\mathrm{IC} 95 \%)^{1}$} & \multirow[t]{2}{*}{ p valor } \\
\hline & $\operatorname{Sim}(\%)$ & Não (\%) & & \\
\hline \multicolumn{5}{|l|}{ Idade da mãe (anos) } \\
\hline 20 à 34 anos & 8,7 & 91,3 & 1,0 & \\
\hline Menos de 15 anos & 15,3 & 84,7 & $1,16(0,96-1,40)$ & 0,126 \\
\hline 16 à 19 anos & 9,3 & 90,7 & $0,61(0,35-1,04)$ & 0,071 \\
\hline Mais de 35 anos & 9,9 & 90,1 & $0,64(0,81-1,40)$ & 0,638 \\
\hline \multicolumn{5}{|l|}{ Escolaridade da mãe } \\
\hline 12 anos ou mais & 8,1 & 91,9 & 1,0 & \\
\hline 9 à 11 anos & 8,7 & 91,3 & $1,21(1,02-1,43)$ & 0,031 \\
\hline Menos de 8 anos & 10,3 & 89,7 & $1,30(1,05-1,62)$ & 0,018 \\
\hline \multicolumn{5}{|l|}{ Estado civil da mãe } \\
\hline Casado & 8,0 & 92,0 & 1,0 & \\
\hline Solteiro & 10 & 89,7 & $1,30(1,13-1,49)$ & 0,001 \\
\hline \multicolumn{5}{|c|}{ Número de consultas de pré-natal } \\
\hline 7 ou mais consultas & 7,8 & 92,2 & 1,0 & \\
\hline 1a 6 consultas & 18,8 & 81,2 & $3,22(2,80-3,71)$ & 0,001 \\
\hline \multicolumn{5}{|c|}{ Duração da gestação (semanas) } \\
\hline 37 semanas ou mais & 4,3 & 95,7 & 1,0 & \\
\hline Menos de 37 & 63,0 & 37,0 & $37,98(31,86-45,28)$ & 0,001 \\
\hline \multicolumn{5}{|l|}{ Tipo de parto } \\
\hline Vaginal & 8,5 & 91,5 & 1,0 & \\
\hline Operatório & 9,3 & 90,7 & $1,09(0,95-1,26)$ & 0,22 \\
\hline \multicolumn{5}{|l|}{ Sexo } \\
\hline Masculino & 7,9 & 92,1 & 1,0 & \\
\hline Feminino & 10,1 & 89,9 & $1,31(1,14-1,51)$ & 0,001 \\
\hline \multicolumn{5}{|c|}{ Número de filhos nascidos vivos } \\
\hline Menos que 4 filhos & 8,8 & 91,2 & 1,0 & \\
\hline 4 ou mais filhos & 13,6 & 86,4 & $1,62(1,16-2,27)$ & 0,004 \\
\hline \multicolumn{5}{|c|}{ Número de filhos nascidos mortos } \\
\hline Menos que 2 filhos & 8,9 & 91,1 & 1,0 & \\
\hline 2 ou mais filhos & 14,2 & 85,8 & $1,68(0,99-2,86)$ & 0,053 \\
\hline
\end{tabular}


Tabela 2. Análise multivariada de variaveis associadas com o Baixo Peso ao Nascer, Divinópolis, Minas Gerais, 2008-2011.

\begin{tabular}{|c|c|c|c|c|c|}
\hline \multirow[t]{2}{*}{ Covariáveis } & \multirow[t]{2}{*}{ Coeficiente } & \multirow[t]{2}{*}{ p-valor } & \multirow[t]{2}{*}{$\mathrm{OR}^{1}$} & \multicolumn{2}{|c|}{ IC 95\% } \\
\hline & & & & Inferior & Superior \\
\hline \multicolumn{6}{|l|}{ Consulta pré-natal } \\
\hline Mais de 7 consultas & & & 1 & & \\
\hline 1 à 6 consultas & 0,677 & $<0,001$ & 1,97 & 1,64 & 2,35 \\
\hline \multicolumn{6}{|l|}{ Duração da gestação } \\
\hline 37 semanas ou mais & & & 1 & & \\
\hline Menos de 37 semanas & 3.566 & $<0,001$ & 35,39 & 29,37 & 42,64 \\
\hline \multicolumn{6}{|l|}{ Sexo } \\
\hline Masculino & & & 1 & & \\
\hline Feminino & 0,467 & $<0,001$ & 1,59 & 1,34 & 1,90 \\
\hline \multicolumn{6}{|l|}{ Estado civil } \\
\hline Casado & & & 1 & & \\
\hline Solteiro & 0,215 & 0,016 & 1,24 & 1,04 & 1,49 \\
\hline
\end{tabular}

manas de gestação, apresentaram um risco de nascer com baixo peso 35,39 vezes maior quando comparadas com crianças nascidas a termo. Outras variáveis também foram consideradas fatores de risco independentes. Os recém-nascidos do sexo feminino apresentaram 59\% mais chance de ter baixo peso em comparação com os nascidos do sexo masculino. O OR para o BPN em mães com menos de 7 consultas pré-natal na gestação foi de 1,97 e em mães solteiras o OR foi de 1,24.

\section{DISCUSSÃO E CONCLUSÃO}

O peso ao nascer é considerado um importante indicador das condições intrauterinas durante a gestação e é considerado o fator individual de maior influência na saúde e sobrevivência do recém-nascido (3). Esta informação está disponível na base do SINASC, considerado atualmente, uma fonte de dados de qualidade em potencial para a construção de indicadores preditores de nascimentos de risco, mortalidade infantil, problemas de desenvolvimento e riscos de doenças e agravos na infância $(17,19)$. Sobre a qualidade da informação, este estudo mostrou que, para as variáveis analisadas no período, o SINASC apresentou excelente completude de preenchimento da DNV, exceto os campos raça/ cor do recém-nascido e anomalia congênita que apresentaram incompletudes elevadas, corroborando outros estudos $(14,19,20)$. É preciso monitorar o preenchimento da DNV utilizando-se de processos educativos, para todos os profissionais envolvidos, favorecendo o aprimoramento da qualidade da informação e a sua utilização para o monitoramento e o planejamento das ações e serviços de saúde materno-infantil. A qualidade do preenchimento dos campos da declaração deve ser transferida para o cotidiano do 
trabalhador, principalmente do enfermeiro, responsável pela supervisão dos registros da equipe de enfermagem na unidade hospitalar (21). Assim, o controle da DNV, a revisão da qualidade do preenchimento, além das orientações seriam algumas das atribuições gerenciais do enfermeiro.

A prevalência de BPN (9,0\%) identificada mostrou-se semelhante às taxas de Minas Gerais $(9,5 \%)$ e da sua macrorregião Oeste $(9,4 \%)$, porém relativamente maior se comparada com a prevalência no Brasil $(8,4 \%)$ (22). A similaridade também foi observada em outros estudos, que apontaram para a tendência no aumento da taxa de recémnascidos de baixo peso nas regiões brasileiras mais desenvolvidas $(10,11,23,24)$. No Rio de Janeiro, São Paulo e Rio Grande do Sul as prevalências identificadas foram de 9,1, 9,2 e $9,4 \%$, respectivamente (22).

$\mathrm{O}$ aumento de BPN verificado neste estudo e em outras regiões desenvolvidas nos últimos anos pode ser explicado pelo aumento de nascimentos prematuros, evento este que pode estar relacionado com o aumento das interrupções da gestação por cesariana ou indução do parto $(2,25)$. Contudo, as prevalências de BPN ainda são variáveis nas diversas regiões do mundo, com desvantagens para aquelas menos desenvolvidas, devido às condições socioeconômicas desfavoráveis das populações (9). Reconhece-se ainda, que as condições maternas e as condições de gestação e parto podem influenciar negativamente no estado nutricional dos recém-nascidos $(9,10)$.

Entre as variáveis relacionadas às condições de gestação e parto, destaca-se como determinante proximal, a duração da gestação. Mais da metade dos bebês de baixo peso nasceu com menos de 37 semanas. A prematuridade e o BPN estão entre os principais fatores determinantes da mortalidade infantil no Brasil e, apesar de a maioria dos indicadores de saúde no país, mostrar progressos nos últimos anos, a taxa de prematuridade tem demonstrado piora, sendo que seu aumento tem sido relacionado com os avanços na sobrevida de recém-nascidos de baixo peso (2). Neste aspecto, pressupõe-se que a instituição de políticas públicas de saúde direcionada para a organização da atenção integral e humanizada ao recém-nascido esteja impactando nas situações grave, como a sobrevida dos recém-nascidos menores de 30 semanas de idade gestacional ou com peso de nascimento menor de $1.000 \mathrm{~g} \mathrm{(26).}$

Por outro lado, o aumento do número de partos operatórios tem contribuído significativamente para o aumento das taxas de prematuridade e, consequentemente, para maior risco de morbidade perinatal (25). O Brasil tem a maior proporção de cesarianas no mundo e entre 2001 e 2008 essa taxa aumentou de 38 para $48,8 \%$, um valor muito acima do esperado (15\%) pela Organização Mundial de Saúde (2). Em Divinópolis, a proporção de partos operatórios (62\%) chega a ser superior a proporção de Minas Gerais (54\%) e do Brasil (52,3\%) e aproxima-se dos índices encontrados na cidade do Rio de Janeiro (57\%) e na cidade de São Paulo (54\%) (22). Contudo, neste estudo os resultados mostraram que o tipo de parto não influenciou a condição de bebês nascidos de baixo peso.

Quanto ao número de consultas de prénatal observou-se que mulheres que realizaram menos de sete consultas apresentaram 97\% mais risco de terem filhos com BPN. Resultados semelhantes foram verificados em vários estudos $(9,27,28)$. Destaca-se que a qualidade e quantidade de cuidados prénatais permitem a identificação de inúmeros fatores e comportamentos de risco, além das complicações decorrentes da gravidez (10). Ao identificar situações de riscos, é possível direcionar intervenções oportunas que previnem desfechos desfavoráveis para a mãe ou para o bebê, além da possibilidade de mantê-los vinculados aos serviços, para seguimentos posteriores, quando a mãe será orientada quanto à importância do auto-cuidado e adoção de hábitos de vida saudáveis. 
A análise multivariada mostrou que as variáveis idade e escolaridade materna, já consagradas como determinantes do BPN $(9,29)$ não apresentaram associação com tal desfecho, corroborando outros estudos $(30$, 31). Entretanto, pode-se observar que a prevalência de BPN foi maior entre mães adolescentes menores de 15 anos. Em relação ao grau de instrução materna verificou-se taxas menores de BPN em mães com maior escolaridade, podendo pressupor que o nível de instrução da mãe, garante acesso aos serviços de pré-natal de melhor qualidade e maternidades mais bem equipadas, oportunizando maior taxa de sobrevida aos seus filhos (11).

$\mathrm{Na}$ análise, também observou-se o efeito do estado civil das mães no BPN. Esses dados são concordantes com os de outros estudos $(28,29)$. Estudo realizado no município de São Luiz averiguou que a ausência de companheiro influencia a adesão das gestantes à assistência pré-natal, o que consequentemente, caso não haja adesão, o monitoramento dos efeitos indesejáveis à mãe e ao recém-nascido ficará comprometido (32).

Observou-se também associação entre BPN e recém-nascidos do sexo feminino, confirmando outros achados (9-11, 28). Crianças do sexo feminino apresentam menor peso do as do sexo masculino de mesma idade gestacional e maior risco de restrição de crescimento intrauterino (28).

Em relação ao número de filhos tidos vivos, apesar de apresentar-se associada ao BPN na análise univariada, quando ajustada a partir da análise multivariada, apurou-se que a paridade foi independente para o BPN.

A tendência ascendente do BPN em Divinópolis esteve relacionada com a duração da gestação, o número de consultas de pré-natal, o sexo feminino e o estado civil da mãe. Os efeitos, prematuridade e inadequação no acompanhamento de pré-natal, apontam para fragilidades na rede de assistência em saúde, em todos os níveis de atenção. A assistência pré-natal apropriada deve ser uma prioridade, a fim de prevenir não só o $\mathrm{BPN}$, mas, outros agravos, como a gravidez na adolescência, que neste estudo representou $11,2 \%$ do total de nascimentos. Destaca-se ainda, o percentual elevado de partos operatórios $(62,0 \%)$ no município, refletindo a condição de desenvolvimento da região.

Como limitação do estudo destaca-se a ausência de outros determinantes socioeconômicos na DNV, e a incompletude dos campos raça/cor do recém-nascido e anomalia congênita, impossibilitando a análise dessas variáveis. Estudar os fatores de risco do BPN e suas implicações para a saúde da criança favorece a disseminação do conhecimento sobre a epidemiologia deste agravo para as diversas áreas da saúde, subsidiando os profissionais para uma assistência qualificada do pré-natal colaborando para a diminuição de fatores de risco modificáveis.

Pressupõe-se que a apropriação desse conhecimento pelos órgãos competentes assegurará o direito de decidir na proporção da necessidade e para aqueles em situação de maior vulnerabilidade e risco de agravos à saúde.

\section{REFERÊNCIAS}

1. Kleinert S, Horton R. Brazil: towards sustainability and equity in health. Lancet [Internet]. 2011 [citado 20 jan 2013]; 377 (9779): 1721-22. Disponível em: http:// download.thelancet.com/flatcontentassets/pdfs/brazil/brazilporcom1.pdf

2. Victora CG, Aquino EML, Leal MC, Monteiro CA, Barros FC, Szwarcwald CL. Maternal and child health in Brazil: progress and challenges. Lancet [Internet]. 2011[citado 22 jan 2013]; 377(9780): 1863-76. Disponível em: http://download.thelancet.com/flatcontentassets/pdfs/ brazil/brazilpor2.pdf

3. Organización Mundial de la Salud. Promoción del desarrollo fetal óptimo. Informe de una reunión consultativa téc- 
nica [Internet]. Ginebra: Organización Mundial de la Salud; 2003 [citado 17 jan 2013]. Disponível em: http://www.who. int/nutrition/publications/fetal_dev_report_ES.pdf

4. Tabrizi FM, Saraswathi G. Maternal anthropometric measurements and other factors: relation with birth weight of neonates. Nutr Res Pract. 2012; 6(2): 132-7.

5. The United Nations Children's Fund (UNICEF). The state of the world's children 2004 [Internet]. New York: UNICEF; 2004 [citado 22 jan 2013]. Disponível em: http://www.unicef.org/sowc04/ files/SOWC_O4_eng.pdf

6. United Nations Children's Fund and World Health Organization. Low Birthweight: Country, regional and global estimates. New York: UNICEF; 2004. 31 p.

7. Ministério da Saúde (BR). Sistema de Informações sobre nascidos vivos [Internet] [citado 17 jan 2013]. Disponível em: www.datasus.gov.br

8. Noronha GA, Lima MC, Lira PIC, Veras AMCA, Gonçalves FCLSP, Filho MB. Evolução da assistência materno-infantil e do peso ao nascer no Estado de Pernambuco em 1997 e 2006. Cien Saude Colet. 2012; 17(10): 2749-56.

9. Carniel EF, Zanolli ML, Antônio MARGM, Morcillo AM. Determinantes do baixo peso ao nascer a partir das Declarações de Nascidos Vivos. Rev Bras Epidemiol. 2008; 11(1): 169-79.

10. Moraes AB, Zanini RR, Riboldi J, Giugliani ERJ. Risk factors for low birth weight in Rio Grande do Sul State, Brazil: classical and multilevel analysis. Cad Saude Publica. 2012; 28(12): 2293-305.

11. Viana KJ, Taddei JAA, Cocetti M, Warkentin S. Peso ao nascer de crianças brasileiras menores de dois anos. Cad Saude Publica. 2013; 29(2): 349-56.

12. Guimarães EAA, Hartz ZMA, Loyola Filho A, Meira AJ, Luz ZMP. Avaliação da implantação do Sistema de Informação sobre Nascidos Vivos em municípios de Minas Gerais, Brasil. Cad Saude Publica. 2013; 29(10): 2105-18.

13. Ministério da Saúde (BR). Manual de instrução para o preenchimento da Declaração de Nascido Vivo. Brasília (DF): Ministério da Saúde; 2011.

14. Jorge MHP, Laurenti R, Gotlieb SLD. Análise da qualidade das estatísticas vitais brasileiras: a experiência de implantação do SIM e do SINASC. Cien Saude Colet. 2007; 12(3): 643-54.

15. Medronho et al. Epidemiologia. São Paulo: Ed. Atheneu, 2006. 493 p.

16. Frias PG, Pereira DMH, Vidal SA, Lira PIC. Avaliação da cobertura do Sistema de Informações sobre Nascidos Vivos e a contribuição das fontes potenciais de notificação do nascimento em dois municípios de Pernambuco, Brasil. Epidemiol. Serv. Saúde. 2007; 16(2): 93-101.

17. Luquetti DV, Koifman RJ. Quality of reporting on birth defects in birth certificates: case study from a Brazilian reference hospital. Cad Saude Publica. 2009; 25(8): 1721-31.

18. Mascarenhas MDM, Gomes KRO. Confiabilidade dos dados do sistema de informações sobre nascidos vivos em Teresina, Estado do Piauí, Brasil, 2002. Cien Saude Colet. 2011; 16(1): 1233-9.

19. Barbuscia DM, Rodrigues-Júnior AL. Completude da informação nas Declarações de Nascido Vivo e nas Declarações de Óbito, neonatal precoce e fetal, da região de Ribeirão Preto, São Paulo, Brasil, 2000-2007. Cad Saude Publica. 2011; 27(6): 192-200.

20. Romero DE, Cunha CB. Avaliação da qualidade das variáveis epidemiológicas e demográficas do sistema de informações sobre nascidos vivos, 2002. Cad Saude Publica. 2007; 23(3): 701-14.

21. Silva GFS, Aidar T, Mathias TAF. Qualidade do sistema de informações de nas- 
cidos vivos no Estado do Paraná, 2000 a 2005. Rev Esc Enferm USP. 2011; 45(1): 79-86.

22. Rede Interagencial de Informações para a Saúde/RIPSA. Indicadores básicos para a saúde no Brasil: conceitos e aplicações. Brasília (DF): Organização Pan-Americana da Saúde; 2010.

23. Andrade CLT, Szwarcwald CL, Castilho EA. Baixo peso ao nascer no Brasil de acordo com as informações sobre nascidos vivos do Ministério da Saúde, 2005. Cad Saude Publica. 2008; 24(11): 256472.

24. Veloso HJ, Silva AA, Barbieri MA, Goldani MZ, Lamy Filho F, Simões VMF, et al. Tendência secular da taxa de baixo peso ao nascer nas capitais brasileiras de 1996 a 2010. Cad Saude Publica. 2013; 29(1): 91-101.

25. Villar J, Carroli G, Zavaleta N, Donner A, Wojdyla D, Faundes A, et al. Maternal and neonatal individual risks and benefits associated with caesarean delivery: multicentre prospective study. BMJ [Internet]. 2007 [citado 20 jan 2013]; 335(1025): 1-11. Disponível em: http://www.bmj. com/content/335/7628/1025

26. Portaria n. 930 (BR). Define as diretrizes e objetivos para a organização da atenção integral e humanizada ao recém-nascido grave ou potencialmente grave e os critérios de classificação e habilitação de leitos de Unidade Neonatal no âmbito do Sistema Único de Saúde (SUS). Ministério da Saúde. Diário Oficial da União [periódico na internet]. 10 mai 2012 [citado 20 Jan 2013]. Disponível em: http://bvsms. saude.gov.br/bvs/saudelegis/gm/2012/ prt0930_10_05_2012.html

27. Heaman M, Newburn-Cook CV, Green CG, Elliott LJ, Helewa ME. Inadequate prenatal care and its association with adverse pregnancy outcomes: A comparison of indices. BMC Pregnancy Childbirth [Internet]. 2008 [citado 22 jan 2013]; 8(15): 1-8. Disponível em http://www. biomedcentral.com/1471-2393/8/15

28. Maia RR, Souza JM. Fatores associados ao baixo peso ao nascer em Município do Norte do Brasil. Rev. bras. cres. desenv. hum. 2010; 20(3): 735-74.

29. Uchimura TT, Pelissari DM, Soares DF, Uchimura NS, Santana RG, Moraes CM. Fatores de risco para o baixo peso ao nascer segundo as variáveis da mãe e do recém-nascido, em Maringá - PR, no período de 1996 a 2002. Cien Cuid Saude. 2007; 6(1): 51-8.

30. Suzuki CM, Ceccon MEJ, Falcão MC, Vaz FAC. Análise comparativa da frequência de prematuridade e baixo peso entre filhos de mães adolescentes e adultas. Rev. bras. cres. desenv. hum. 2007; 17(3): 95103.

31. Tiago LF, Caldeira AP, Vieira MA. Fatores de risco de baixo peso ao nascimento em maternidade pública do interior de $\mathrm{Mi}$ nas Gerais. Pediatria (São Paulo). 2008; 30(1): 8-14.

32. Coimbra LC, Silva AAM, Mochel EG, Alves MT, Ribeiro VS, Aragão VMF, et al. Fatores associados à inadequação do uso da assistência pré-natal. Ver Saude Publica. 2003 ; 37(4): 456-62. 